\title{
AMNIOTIC MEMBRANE TRANSPLANTATION - EXCELLENT BIOLOGICAL SCAFFOLD FOR ANTERIOR OCULAR SURFACE RECONSTRUCTION
}

\author{
Yana Manolova' ${ }^{1}$ Cvetan Popov ${ }^{2}$, Christina Grupcheva ${ }^{1}$ \\ ${ }^{1}$ Department of Ophthalmology and Visual Science, Medical University of Varna \\ ${ }^{2}$ Center for Translational Medicine and Cell Therapy - Varna, Bulgaria
}

\begin{abstract}
AIM: To evaluate the clinical effect and to determine the correlation between the initial pathology and postoperative outcome in patients with anterior surface dysfunction treated by amniotic membrane transplantation (AMT).

MATERIALS AND METHODS: 174 AMT of cryopreserved amniotic membrane (AM) were performed on 128 patients over a period of two years (January, 2014- December, 2015). Patients were grouped into 7 cohorts according to the reasons for the AMT - A) corneal recurrent erosions $(n=32), B)$ postoperative keratitis $(n=7), C)$ neurotrophic ulcers $(n=14), D)$ trauma and burns $(n=30), E)$ bacterial keratitis $(n=10)$, F) viral keratitis $(n=19)$ and $G)$ other $(n=16)$. The groups were assessed for levels of pain (a subjective symptom) and hyperemia and size of the corneal ulcer (objective signs), and were compared on several stages before and after AMT - day 0, day 8 and day 25.

RESULTS: The AM treatment resulted in significant reduction of the symptoms in all categories. On the $8^{\text {th }}$ day, the observed improvement was higher than $50 \%$ (100\% for the pain, $50 \pm 10 \%$ for hyperemia and $60 \pm 15 \%$ for corneal ulcer) as at the end of our follow-up hyperemia improvement increased to $80 \pm 10 \%$ and corneal ulcer size decreased with $95 \pm 3 \%$. Interestingly, in comparison to day 8 , pain levels increased significantly after 25 days of AMT to $15 \pm 15 \%$.

CONCLUSION: AMT is an effective method for managing pathological conditions resulting from a damage to the ocular surfaces such as unresponsiveness to common treatment, chronical or recurrent diseases, eye infection and stem cell deficiency. The application of this therapy can have permanent results, which lead to complete healing of the damaged eye or it can serve as a temporal procedure until reconstructive approach is possible. AMT is a treatment of choice in the cases where availability of corneal tissue is limited.
\end{abstract}

Keywords: human amniotic membrane, transplantation, pain, hyperemia and corneal ulcer

\author{
Address for correspondence: \\ Yana Manolova \\ Specialized Eye Hospital \\ 15 Doyran Str. \\ Varna \\ e-mail:y.m.manolova@gmail.com
}

Received: September 7, 2016

Accepted: September 21, 2016

\section{INTRODUCTION}

The eyes allow us to perceive, orientate and navigate, but at the same time are in constant exposure to the dangers of the surrounding environment. Thus, nature has developed several protective mechanisms such as bony orbits, eyelids, tear film, strong corneal and conjunctival epithelium, and a submucosal secretory immunoglobulin system (1). Howev- 
er, often due to different factors such as disease, inflammation or chemical burn, these eye-protecting mechanisms are not sufficient and patients develop ocular surface disorders (OSDs). They commonly result in progressive inflammation, vascularization, scarring or even loss of visual function, when not properly treated.

The gold standard in ophthalmology for treatment of OSDs for the last two decades is transplantation of human amniotic membrane (AM). Historically, AM was used first by de Rötth (2), however, its real potential was recognized in the middle of the nineties after the publication of Kim and Tseng (3). They demonstrated that the damaging effect of treating New Zealand albino rabbit eyes with n-heptanol was only overcome by the experimental group, subjected to AM treatment. Moreover, they observed that their eyes became clear with either minimal or no vascularization (3).

The advantage of using AM for enhancing the healing of the human eyes comes from its similarity to the corneal and conjunctival tissue and its anti-inflammatory, anti-scarring and anti-angiogenic activity, and most importantly from their ability to promote epithelialization $(4,5)$. These advantages allow the tissue to be used as a filling material (6) or as a surface cover that protects the damaged eye from the surrounding environment (7). AM is the innermost layer of the placenta and forms the amniotic cavity. The membrane is composed of several layers including a single layer of epithelial cells, basement membrane and avascular connective tissue matrix (7). The main protein components of AM are the collagens (types IV, V, and VII), however, it also contains fibronectin, laminins, proteoglycans and glycosaminoglycans. AM possesses a number of important growth factors - epidermal growth factor (EGF), transforming growth factor beta (TGF- $\beta$ ), fibroblast growth factor (FGF), and platelet-derived growth factors (PDGF), which support its trophic function. Moreover, it has no HLA-A, B, C antigens and $\beta 2$ microglobulin that leads to graph-host reaction (4). All these advantages make the AM the gold standard in the treatment of OSDs such as persistent epithelial defects, corneal ulcers, descemetocele formation, corneal perforation, limbal stem cell deficiency, symptomatic bullous keratopathy, band keratopathy, chemical injury, thermal injury, scleral perforation and etc.(9). Furthermore, recently AM has been used also as a scaffold for ex vivo cultivation of limbal, corneal and conjunctival epithelial cells (10).

Despite the common use of AM in ophthalmology for the healing of human eyes, there are very few studies, describing the effect of successful AMT by targeting the most prominent subjective symptom pain and the clinical signs - hyperemia and size of corneal ulcer. Thus, the aim of our study was to evaluate the changes of pain and pain release as a consequence to the AMT and to investigate the postoperative effect onto the clinical signs in seven different groups that combine the major ophthalmological diseases.

\section{MATERIALS AND METHODS}

Patients

In this study we performed 174 AMT on 128 patients (several patients received more than one AMT). Among them, 69 patients were male and 59 female. Regarding the age of our patients, the medium age was 57.6 years as the patients over $51(n=87)$ had the highest number, followed by patients in the age group of 33-50 years $(n=33)$ and just several patients were younger than 30 years $(n=8)$. Based on their history and clinical signs, they were separated into seven cohorts - A) corneal recurrent erosions $(n=32), B)$ postoperative keratitis $(n=7), C)$ neurotrophic ulcers $(n=14), D)$ trauma and burns $(n=30)$, E) bacterial keratitis $(n=10), F)$ viral keratitis $(n=19)$ and $G$ ) other $(n=16)$. In order to identify the effects of AMT, the subjective and the objective clinical symptoms were examined before surgery and on days 8 and 25 after AMT.

\section{Preoperative Examination}

In accordance with the Good Clinical Practice in Ophthalmology, the preoperative examination of the patients included assessment of subjective signs pain, photosensitivity, redness, tearing, anterior ocular surface and clinical signs such as hyperemia, neovascularization, size of corneal ulcer, presence of infiltration, hypopyon and in vivo laser-scanning confocal microscopy (LSCM). In order to scale the different parameters, we graded them on a scale from 1 to 5 . For the pain classification: grade 1 means no pain, grade 2 - somewhat painful, grade 3 - constant mild pain, grade 4 - moderate to severe pain and grade 5 - severe to extreme pain. For hyperemia: 
grade 1 meant absent, grade 2 was mild, grade 3 - average, grade 4 - moderate, 5 - severe. The corneal ulcer size was classified as follows - grade $1-<0.5 \mathrm{~mm}$, grade 2 - $0.5-1.0 \mathrm{~mm}$, grade $3->1.0-2.0 \mathrm{~mm}$, grade 4 $>2.0-5.0 \mathrm{~mm}, 5->5.0 \mathrm{~mm}$ ). For the observation of the anterior ocular surface and anterior ocular segment we used Reichert XCEL 400 slit lamp. Measuring of the corneal thickness was performed with 3D anterior segment optical coherence tomography (AS-OCT; Topcon 2000) with lateral resolution of $\leq 20 \mu \mathrm{m}$ and in-depth resolution of 5-6 $\mu \mathrm{m}$.

\section{Amniotic Membrane}

The use and handling of the AM for transplantation was according to Tissue Bank Bioregeneration-Sofia. All AMs were tested negative for HIV, Hepatitis B and C, and syphilis.

Amniotic Membrane Transplantation - Surgical Technique

The AM transplantation was done as follows: 1) the AM was pre-warmed at room temperature; 2) the patient's cornea epithelium was removed and the Bauman's membrane was polished; 3) the AM was washed (to remove the glycerine); in cases with epithelial defects without stromal thinning a single layer of membrane with epithelial side down was used to cover the defect by trimming off the excess parts and suture with 8-0 Vicryl treed; in cases with stromal thinning two layers of amniotic membrane were placed; the first membrane filled the defect as it was placed with the epithelial side up and second covers the cornea with epithelial side down and was sutured with 8-0 Vicryl suture; 4) on the top of the membrane (regardless of which approach we used) we applied bandage/therapeutic contact lens (Pure Vision 2, Pure Vision HD).

\section{Postoperative Treatment and Follow-up}

\section{Examination}

After the surgery all patients received topical therapy with steroid drops, non-steroid anti-inflammatory drops, antibiotics drops and lubrication. Upon healing, the antibiotics were discontinued; however, topical steroid, non-inflammatory drops and lubrication were kept as part of the regimen prescribed to the patients. The assessment of the surgical outcome was determined by the patient's subjective (pain) and several objective signs (hyperemia and size of the corneal ulcer) with the help of slit lamp and photo documentation at the defined observation points - on postoperative day 1 , at the end of the first week (day 8) and the end of the fourth week (day 25).

\section{Statistical Analysis}

Quantitative data was analyzed with GraphPad Prism 5 software (GraphPad, La Jolla, USA). Bar charts show mean values and standard deviations. For measuring the significance we used ANOVA on RANKS test with Student-Newman-Keuls Method and p-value below $0.05 \%$ was considered statistically significant. Significance was indicated in the figures as follows ${ }^{*} \mathrm{p}<0.05,{ }^{* *} \mathrm{p}<0.005,{ }^{* *} \mathrm{p}<0.0005$.

\section{RESULTS}

An improvement of all three symptoms was detected in all seven groups after 1 month post AMT. We found that at day 8 the pain was almost completely diminishing in all groups (Fig. 1). Interestingly, we found that the patient group $\mathrm{C}$, which is the neurotrophic group, had initially very low levels of pain. However, also here we observed an improvement of this factor on day 8 , which was persistent also on day 25 . Among the rest of the groups, higher pain was felt by group $\mathrm{F}$ (bacterial), group D (trauma and burns) and group $\mathrm{G}$ (viral), followed by group B (post-surgical), group $\mathrm{E}$ (others) and group A (persistent corneal defects). However, the differences between these six groups were not significant. Pain relief was better expressed in the patients with viral keratitis and causes with persistent corneal defects, the levels of which increased the least on day 25 improving the levels by $74 \%$ and $72 \%$, correspondingly. In the other four groups, despite the increased level of pain on day 25 , their value remained under $30 \%$ of the initially experienced by the patient pain. The worst pain remained in group $\mathrm{F}$ (bacterial keratitis), which was approximately $40 \%$ of the initial pain.

Analysis of the two clinical factors (objective signs) - hyperemia and size of the neurotrophic ulcers demonstrates a similar pattern. We clearly show that upon application of AM the level of hyperemia and the size of the neurotrophic ulcers reduce over time as they almost completely disappear on day 25 . Initially, we observed different levels of hyperemia in our groups as the highest level of hyperemia we saw in the groups D (trauma and burns), C (neurotrophic ulcers) and $\mathrm{F}$ (bacterial keratitis), followed by group E (others), B (post-surgical) and the lowest lev- 


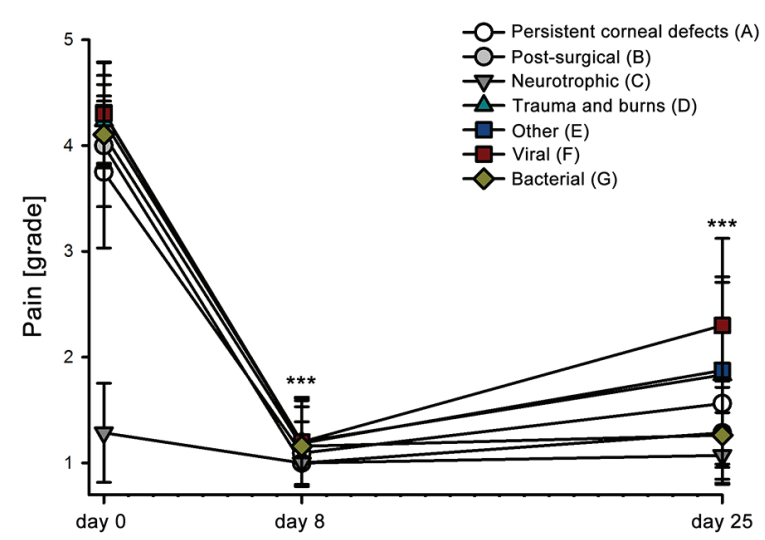

Fig. 1. Decreasing levels of pain upon AMT. The graph shows significant pain release on day 8 after AMT. However, we observed an increase of the pain in the patients on day 25. Grades: 1- no pain, 2 - somewhat painful, 3 constant mild pain, 4 - moderate to severe pain and 5 severe to extreme pain. Statistical significance: ${ }^{\star} p<0.05$,

$$
{ }^{* *} p<0.005,{ }^{* * *} p<0.0005 \text {. }
$$

el of hyperemia was observed in group A (persistent corneal defects) (Fig. 2). Upon membrane application, the levels of hyperemia significantly decreased in all groups on day 8 and especially on day 25 . At the end of the followed period we observed at least a twofold decrease of the hyperemia, as the decrease varied based on the initial level in the groups. On day 25 we found that in-between the groups the distribution was similar to day 0 (before the AMT) as higher levels of hyperemia remained in groups G, E, D, and F, followed by $\mathrm{D}$ and finally $\mathrm{A}$. Nonetheless, the differences between the groups were not very pronounced and often their values overlapped.

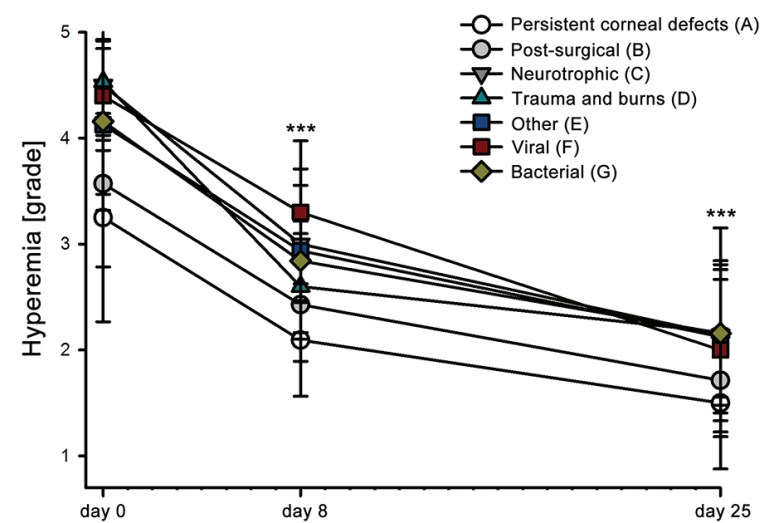

Fig. 2. Decreasing levels of hyperemia after AMT. The graph shows significant reduction of the hyperemia on day 8 and day 25. Grades: 1 - absent, 2 - mild, 3 - average, 4 - moderate and 5 - severe. Statistical significance: ${ }^{*} p<0.05,{ }^{* *} p<0.005,{ }^{* * *} p<0.0005$.
The size of the neurotrophic ulcers before application of AMT demonstrated heterogeneous pattern (Fig. 3). Here the ulcer size was significantly bigger in group $\mathrm{E}$ (above $5.0 \mathrm{~mm}$ ), followed closely by groups $\mathrm{F}, \mathrm{B}, \mathrm{C}$, and D (with average of 2.0-5.0 $\mathrm{mm}$ ). There was significant difference in the other two groups $\mathrm{G}$ and $\mathrm{A}$, where the ulcers had an average size of 1.0$2.0 \mathrm{~mm}$ and $0.5-1.0 \mathrm{~mm}$, correspondingly. However, upon application of the AMT, we observed a decrease of the ulcer size in all seven groups on day 8 and significant difference on day 25 . On day 25 , in all the investigated groups the size of neurotrophic ulcers was close to $0.5 \mathrm{~mm}$ and there were no significant differences in the investigated groups, which clustered together.

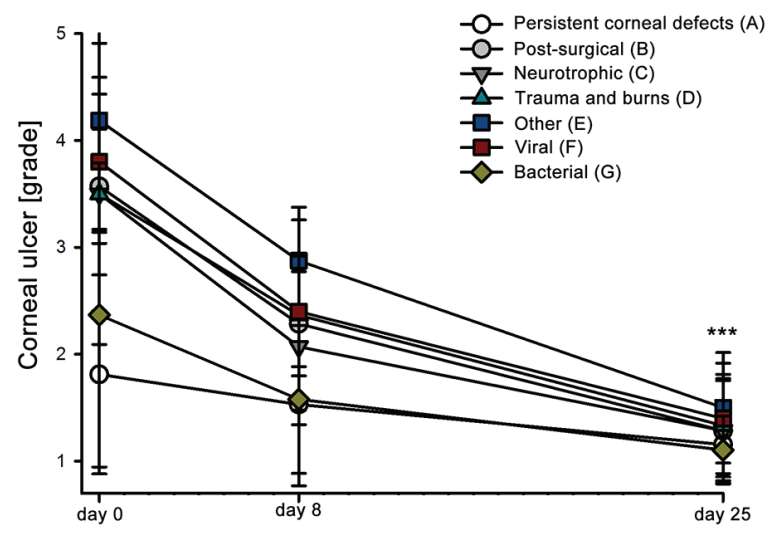

Fig. 3. Changes in the size of corneal ulcers upon AMT. The graph show significant decrease of the ulcer size at day 8 after AMT in groups F, B, C and D. At day 25 all groups clustered together as their average size was approx. $0.5 \mathrm{~mm}$. Grades: 1-<0.5 mm, 2- 0.5-1.0 mm, 3- >1.0-2.0 $\mathrm{mm}, 4->2.0-5.0 \mathrm{~mm}$ and 5- $>5.0 \mathrm{~mm}$. Statistical significance: ${ }^{*} p<0.05,{ }^{* *} p<0.005,{ }^{* *} p<0.0005$.

An example of the significant improvement resulting of application of AM was demonstrated in Fig. 4. The patient suffered traumatic event was photo documented at the first day upon trauma (Fig. 4A and B) and 10 days after medication treatment (Fig. 4C). We observed no significant improvement of the damaged cornea upon drug application even after 17 days of treatment and furthermore, we detected a formation of multiple marginal infiltrates in the eye (Fig. 4D). Therefore, we apply AM to the site of injury (Fig. 4E) and observed that after AMT the defect was greatly reduced as just one marginal infiltrate left at 
the end of first (Fig. 4F) and third month of treatment (Fig. 4G). Finally, our patient recovers its vision as the visual indicator for BCVA was 20/20.

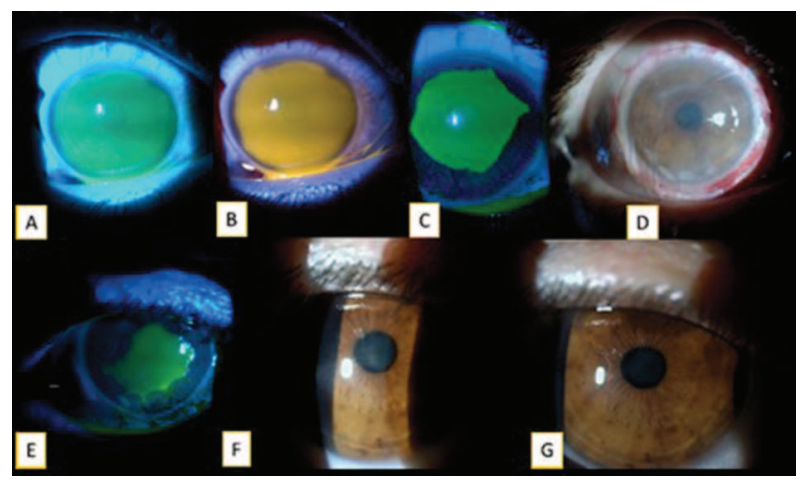

Fig. 4. Effect of AMT application on a patient who suffered a traumatic event. A and B) Photo documentation on the first day of the trauma; C) 10 days of medication treatment; D) 17 days of medication treatment, we detected a formation of multiple marginal infiltrates in the eye; application of AM on the eye; E) after the AMT- the defect is greatly reduced and just one marginal infiltrate is left; F) 1 month after AMT; G) 3 months after AMT.

\section{DISCUSSION}

The aim of our study was to evaluate the importance of AMT for the proper healing of the damaged anterior ocular surface. We clearly demonstrated here that application of AM have a positive effect on the healing process of the damaged eye and its application is indispensable for the reduction of prominent subjective symptoms like pain and clinical signs such as hyperemia and the size of the corneal ulcer. However, we here suggest that the basic treatment of damaged anterior ocular surface should consist of a combination of medical treatment and transplantation of amniotic membrane.

The results of present studies highlight the important role of the AM for the treatment of anterior ocular surface damage, often associated with delayed recovery and risk of intraocular inflammation. Important part of the discomfort caused by eye damage is the pain, which is the major social issue that influences the quality of life. AM acts as a biological bandage that protects the regenerating epithelium from friction forces, caused by the blinking movement of the eyelids. Furthermore, it also significant- ly relieves pain, due to its adhesion to injury surface and covering of the neural endings $(11,12)$. AM also protects the injury surface from drying, which further increases the sense of pain in the patients. Similar results regarding the pain relief were observed by others $(13,14,15,16)$. Tok et al., 2015 (13) demonstrated in fifteen eyes of 10 patients with abuse of topical anesthetics that the initial medical treatment was not effective due to severe pain, persistent epithelial defects and progressing stromal thinning. Therefore, the authors performed AMT, which resulted in a significant improvement in all but two causes. In another publication, Siu et al., 2015 (14) examined a total of 21 eyes of 20 bullous keratopathy patients and measured the mean pain score pre-operatively and post-operatively in regard to the AMT. They found that post-operatively $76 \%$ of the eyes were completely pain-free, while the rest experienced minor pain and $47 \%$ of the eyes remained symptom-free for up to 36 months. The results of our study were in line with the literature. We observed a significant decrease of pain immediately after the application of the AM. Interestingly, on day 25 in some groups the levels of pain increased. We speculated that this small increase of the pain felt by the patients was due to the dissolving of the AM transplant (up to two weeks), which resulted in a removal of the anti-inflammatory, anti-scarring and antiangiogenic effects of the membrane, and in an exposure of the eye to the environment. Nonetheless, the changes between the groups on day 8 and day 25 were not severe and the level of pain on day 25 still remained similar to that of day 0 . Altogether, others and our work demonstrated that the application of the AM is essential for decreasing the pain in patients with damaged anterior ocular surface.

In addition to the pain relief, it was reported that AMs have a positive effect on the inflammatory processes in the eye such as hyperemia and corneal ulcer. Prior to 2014, the exact mechanism of the antiinflammatory properties of AM was not completely understood. It was suggested that AM acts as a barrier and decreases the influx of inflammatory cells to the site of injury $(16,17,18,19)$. Later it was discovered that the epithelium of the amniotic membrane can inhibit the chemotactic activity of neutrophils and macrophages, T- and B-cells proliferation and further on it can induce apoptosis in them without af- 
fecting the epithelial cells of the cornea (20). In 2014 it was discovered that the observed anti-inflammatory effect of AM was due to a complex, composed of hyaluronic acid and protein called pentraxin 3 (HCHA/ PTX3) (19,20). This unique matrix complex was first derived from human AM and besides exerting anti-inflammatory, anti-scarring, and antiangiogenic effects, the HC-HA/PTX3 complex can also maintain limbal niche cells by supporting the quiescence of limbal epithelial stem cells $(21,22,23,24,25)$.

For centuries, we clinicians have recognized that ocular hyperemia is a hallmark of several ophthalmic pathologies. As with any inflammatory reaction, the hyperemia on the ocular surface is a result of a tissue injury that lead to an inflammatory response due to increased blood flow and leakage of proteins and fluids from the capillaries, which can cause edema and tissue damage. Application of AM was verified to have a positive effect on patients with hyperemia (26,27). For example, Huang et al., 2005 (26) demonstrated that patients who underwent Pterygium resection plus amnion transplantation recovered faster than the groups without AMT and had lower levels of hyperemia of conjunctiva and irritation symptoms in the eye. Similarly, Cheng et al., 2016 (28) showed that application of self-retained cryopreserved AM in patients with dry eye can significantly reduce the conjunctival hyperemia for an average of 5 days and preserve the symptom-free condition for up to 4 months. Our results were in line with the published data, as we observed constant significant decrease of the hyperemia upon AMT. Others and our results strongly support the proposed positive effect of AM application for reduction of hyperemia of the eyes.

Another factor, indicator for an ongoing inflammation of the anterior ocular surface is formation of corneal ulcers. A corneal ulcer is a break in the outer epithelial layer of the cornea that fails to heal and is often caused by infection (29). Corneal ulcer, or ulcerative keratitis, is an inflammatory or more seriously, infective condition of the cornea involving disruption of its epithelial layer with an involvement of the corneal stroma. Healing of this condition can be achieved by ATM. There are numbers of publications demonstrating that application of AM lead to improved or complete healing of the corneal ulcers $(30,31,32,33,34)$. Again, our study confirmed the literature observations, as we reported here, that application of AM to our seven groups, which includes the major ophthalmological diseases, can severely improve the size of a corneal ulcer.

To our knowledge, this study is the first one investigating in parallel the effect of AM application onto seven different groups that combine the major ophthalmological diseases. We confidently demonstrated in our patient cohort that despite the disease, AMT can successfully improve the most prominent subjective symptom - pain and the inflammation clinical signs such as hyperemia and size of corneal ulcer. Therefore, we conclude that the effect observed by $\mathrm{AM}$ is not disease-related, but rather a common function of the amniotic membrane and we strongly recommend considering the use of AMT for treatment in any of the discussed in this article anterior ocular surface diseases.

\section{CONCLUSION}

Taking into account the similarity to the cornea and its anti-inflammatory, anti-scarring, and antiangiogenic effects, it is understandable why AMT is the gold standard for dealing with difficult to heal pathologies of the anterior ocular surface. Here we demonstrated that together with these positive effects, application of AM lead to a significant pain relief, reduction of the levels of hyperemia and the size of the corneal ulcers. Moreover, it also enhanced the recovering of the anterior ocular surface and often omitted the need of prolonged use of expensive medications, which was often demonstrated in the literature.

\section{REFERENCES}

1. Lemp M. A. and Blackman H. J.. Ocular Surface Defense Mechanisms. - Annals of Ophthalmology January 1981;13,no.1:61-63.

2. De Rotth A. Plastic repair of conjunctival defects with fetal membranes. - Arch. Ophthalmol. 1940; 23:522-522;

3. Kim J. C. and Tseng S. C.. Transplantation of Preserved Human Amniotic Membrane for Surface Reconstruction in Severely Damaged Rabbit Corneas. - Cornea September 1995;14, no.5:473-84.

4. Antonietta R., Silini et al. The Long Path of Human Placenta, and Its Derivatives, in Regenerative Medicine. - Frontiers in Bioengineering and Biotechnology.2015; 3: 162. 
5. Fernandes M. et al. Outcome of Pterygium Surgery: Analysis over 14 Years. - Eye (London, England ),November 2005;19, no.11:1182-90.

6. Fan Junhua, Wang Meihua, and Zhong Fulu. Improvement of Amniotic Membrane Method for the Treatment of Corneal Perforation. - BioMed Research International 2016.

7. Gheorghe A. et al. New Clinical Application of Amniotic Membrane Transplant for Ocular Surface Disease. - Journal of Medicine and Life June 2016; 9, no.2:177-79.

8. Fetterolf Donald E. and Snyder Robert J.. Scientific and Clinical Support for the Use of Dehydrated Amniotic Membrane in Wound Management. Wounds: A Compendium of Clinical Research and Practice October 2012;24, no.10:299-307.

9. Sangwan Virender S. et al. Amniotic Membrane Transplantation: A Review of Current Indications in the Management of Ophthalmic Disorders. - Indian Journal of Ophthalmology August 2007;55, no.4:251-60.

10. Tseng S.C.G. et al. Critical Appraisal of Ex Vivo Expansion of Human Limbal Epithelial Stem Cells. - Current Molecular Medicine December 2010;10, no. 9:841-50.self renewal and fate decision of limbal SCs are regulated by a specialized in vivo microenvironment, termed "niche". Loss of limbal SCs or dysfunction of the limbal niche renders corneas with a unique clinical disease labeled limbal stem cell deficiency (LSCD

11. Baum Jules. Thygeson Lecture. Amniotic Membrane Transplantation: Why Is It Effective?. - Cornea May 2002;21, no.4:339-41.

12. Lee H. S. and Kim J. C.. Effect of Amniotic Fluid in Corneal Sensitivity and Nerve Regeneration after Excimer Laser Ablation. - Cornea September 1996;15, no.5:517-24.

13. Ozlem Yalcin Tok et al. Toxic Keratopathy Associated with Abuse of Topical Anesthetics and Amniotic Membrane Transplantation for Treatment. - International Journal of Ophthalmology 2015;8, no. 5: 938-44.

14. Gillian D. J. Y. Siu, Alvin L. Young, and Lulu L. Cheng. Long-Term Symptomatic Relief of Bullous Keratopathy with Amniotic Membrane Transplant. International Ophthalmology December 2015;35, no. 6.

15. Shreya Thatte. Amniotic Membrane Transplantation: An Option for Ocular Surface Disorders. -
Oman Journal of Ophthalmology May 2011;4, no. 2: 67-72.

16. Shimmura S. et al. Antiinflammatory Effects of Amniotic Membrane Transplantation in Ocular Surface Disorders, - Cornea May 2001; 20, no. 4:408-13.|luc0|lu8221\{\} \{\i\{\}Cornea\} 20, no. 4 (May 2001

17. Solomon A. et al. Suppression of Interleukin 1alpha and Interleukin 1beta in Human Limbal Epithelial Cells Cultured on the Amniotic Membrane Stromal Matrix. The British Journal of Ophthalmology April 2001; 85, no.4:444-49.

18. Tseng S. C., D. Q. Li, and X. Ma. Suppression of Transforming Growth Factor-Beta Isoforms, TGFBeta Receptor Type II, and Myofibroblast Differentiation in Cultured Human Corneal and Limbal Fibroblasts by Amniotic Membrane Matrix,. - Journal of Cellular Physiology June 1999;179, no.3:32535.TGF-Beta Receptor Type II, and Myofibroblast Differentiation in Cultured Human Corneal and Limbal Fibroblasts by Amniotic Membrane Matrix, |luc0\|u8221\{\} \{\i\{\}Journal of Cellular Physiology\} 179, no. 3 (June 1999

19. Li Haochuan et al. Immunosuppressive Factors Secreted by Human Amniotic Epithelial Cells. - Investigative Ophthalmology \& Visual Science March 2005; 46, no. 3:900-907.

20. Kamiya K, Wang M, Uchida S, Amano S, Osaka T, Sakuragawa N, et al. Topical application of culture supernatant from human amniotic epithelial cells suppresses inflammatory reactions in cornea. - Exp Eye Res 2005; 80:671-679.

21. Wei Li et al. Amniotic Membrane Induces Apoptosis of Interferon-Gamma Activated Macrophages in Vitro. - Experimental Eye Research February 2006; 82, no. 2:282-92.

22. He Hua et al. Suppression of Activation and Induction of Apoptosis in RAW264.7 Cells by Amniotic Membrane Extract. - Investigative Ophthalmology \& Visual Science October 2008; 49, no. 10:4468-75.

23. Zhang Suzhen et al. Constitutive Expression of Inter- $\alpha$-Inhibitor (I $\mathrm{I}$ ) Family Proteins and Tumor Necrosis Factor-Stimulated Gene-6 (TSG6) by Human Amniotic Membrane Epithelial and Stromal Cells Supporting Formation of the Heavy Chain-Hyaluronan (HC-HA) Complex. - The Journal of Biological Chemistry April 2012; 287, no. 15:12433-44. 
24. Tseng Scheffer C. G. HC-HA/PTX3 Purified From Amniotic Membrane as Novel Regenerative Matrix: Insight Into Relationship Between Inflammation and Regeneration. - Investigative Ophthalmology \& Visual Science April 2016; 57, no. 5: ORSFh1-8.

25. Chen Szu-Yu et al. HC-HA/PTX3 Purified From Amniotic Membrane Promotes BMP Signaling in Limbal Niche Cells to Maintain Quiescence of Limbal Epithelial Progenitor/Stem Cells. Stem Cells (Dayton, Ohio) November 2015; 33, no. 11:3341-55.

26. Huang Yan, Wang,Banwei and Ye Qinying. Clinical study in combining application of mitomycin and amnion transplantation together with pterygium resection. Yan Ke Xue Bao = Eye Science / "Yan Ke Xue Bao" Bian Ji Bu December 2005; 21, no. 4: $110-13$.

27. Wanhong Liang, Rongru Li, and Xingyun Deng. Comparison of the Efficacy of Pterygium Resection Combined with Conjunctival Autograft versus Pterygium Resection Combined with Amniotic Membrane Transplantation. Eye Science June 2012; 27, no. 2: 102-5.

28. Cheng Anny M. S. et al. Morselized Amniotic Membrane Tissue for Refractory Corneal Epithelial Defects in Cicatricial Ocular Surface Diseases. Translational Vision Science \& Technology May 2016; 5, no. 3: 9 .

29. Alio L.Jorge, Arnalich-Montiel Francisco, and Rodriguez E. Alejandra. The Role Of 'eye Platelet Rich plasma' (E-PRP) for Wound Healing in Ophthalmology. Current Pharmaceutical Biotechnology June 2012; 13, no. 7: 1257-65.no. 7 (June 2012

30. Morikawa Keisuke et al. Indication and Efficacy of Amniotic Membrane Transplantation Performed under Advanced Medical Healthcare. Nippon Ganka Gakkai Zasshi April 2016; 120, no. 4: 291-95.

31. Uhlig E. Constantin et al. Long-Term Efficacy of Glycerine-Processed Amniotic Membrane Transplantation in Patients with Corneal Ulcer. Acta Ophthalmologica September 2015; 93, no. 6: e481-487.

32. Virender S. Sangwan et al. Amniotic Membrane Transplantation: A Review of Current Indications in the Management of Ophthalmic Disorders. Indian Journal of Ophthalmology August 2007;55, no. 4: 251-60.
33. Prabhasawat P., Tesavibul N., and Komolsuradej W. Single and Multilayer Amniotic Membrane Transplantation for Persistent Corneal Epithelial Defect with and without Stromal Thinning and Perforation. - The British Journal of Ophthalmology December 2001; 85, no. 12:1455-63.

34. Iakimenko A.Stanislav, Buznyk I. Oleksiy, and Rymgayllo-Jankowska Beata. Amniotic Membrane Transplantation in Treatment of Persistent Corneal Ulceration after Severe Chemical and Thermal Eye Injuries. European Journal of Ophthalmology August 2013; 23, no. 4: 496-503.2001 